\title{
Las madres y la publicidad de alimentos dirigida a niños y niñas: percepciones y experiencias
}

\author{
Mothers and food advertising directed at children: \\ perceptions and experiences
}

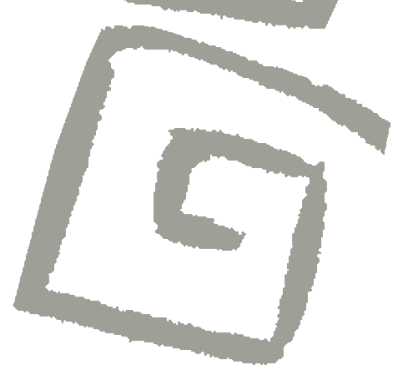

Luciana Castronuovo', Patricia Gutkowski², Victoria Tiscornia ${ }^{3}$, Lorena Allemandi ${ }^{4}$
${ }^{1}$ Doctoranda en Ciencias Sociales. Investigadora, Fundación InterAmericana del Corazón Argentina (FIC) Argentina. $\bowtie$ (iD)

${ }^{2}$ Maestranda en Gestión de la Comunicación de las Organizaciones. Directora del Área de Comunicación, Fundación InterAmericana del Corazón Argentina (FIC), Argentina. $\triangle$ id

${ }^{3}$ Maestranda en Nutrición Humana. Investigadora, Fundación InterAmericana del Corazón Argentina (FIC), Argentina. $\triangle$ iD

${ }^{4}$ Maestranda en Salud Pública. Directora del Área de Alimentación, Fundación InterAmericana del Corazón Argentina (FIC), Argentina. $\Delta$ iD
RESUMEN El objetivo de la investigación es analizar cómo las publicidades de alimentos son percibidas por madres de distintos sectores socioeconómicos del Área Metropolitana de Buenos Aires (AMBA), Argentina. Entre mayo y noviembre de 2015, se realizaron ocho grupos focales en los que participaron un total de 49 madres de la región del AMBA, con distinto nivel educativo. La investigación ha dado cuenta de cómo las decisiones de compra de las madres son afectadas por los pedidos de los niños y niñas impulsados por la publicidad y las promociones. También mostró cómo estas publicidades y promociones se combinan con otros elementos del contexto (mayor oferta de productos, niños "más demandantes") que afectan el proceso de toma de decisiones de las madres respecto a la alimentación de sus hijos y permiten instalar el consumo de determinados productos no saludables. Esta situación se ha observado en el conjunto de madres, sin diferir según el nivel educativo alcanzado.

PALABRAS CLAVES Publicidad de Alimentos; Promoción de Alimentos; Toma de Decisiones; Grupos Focales; Argentina.

ABSTRACT The objective of this study is to analyze how food advertising is perceived by mothers from different socioeconomic sectors of the Metropolitan Area of Buenos Aires, Argentina. Between May and November 2015, eight focus groups were conducted with the participation of 49 mothers of different education levels living in the study area. The results show how the purchasing decisions of mothers are influenced by the requests of their children, which are in turn prompted by food advertising and promotion. The study also shows how food advertising and promotion are combined with other environmental factors (greater supply of food products, "more demanding" children) that affect the decision-making process of mothers regarding their children's nutrition and foster the consumption of certain unhealthy products. This situation was observed in all the focus groups, without differences among education levels.

KEY WORDS Food Publicity; Food Promotion; Decision Making; Focus Groups; Argentina. 


\section{INTRODUCCIÓN}

La Organización Mundial de la Salud (OMS) considera que el sobrepeso y la obesidad infantil constituyen uno de los principales problemas de salud pública del siglo XXI. Según sus estimaciones, en 2010 existían en el mundo alrededor de 42 millones de niños y niñas menores de cinco años con sobrepeso u obesidad, de los cuales casi 35 millones vivían en países en desarrollo ${ }^{(1)}$.

De acuerdo a la Organización de las Naciones Unidas para la Agricultura y la Alimentación (FAO), en los últimos años se dio un proceso conocido como la "doble carga de la malnutrición" a partir del cual comenzaron a convivir la desnutrición infantil y un aumento considerable de la prevalencia de obesidad ${ }^{(2)}$. Este crecimiento mundial de los índices de sobrepeso y obesidad en los niños y niñas se debe a una multiplicidad de factores: el cambio en los patrones de consumo que implicó un aumento de la ingesta de alimentos industrializados de bajo valor nutritivo, ricos en grasas, azúcares y sal; la baja accesibilidad a alimentos de alta calidad nutricional, como frutas y verduras; $y$ el aumento del sedentarismo ${ }^{(3)}$.

En Argentina, según la última Encuesta Mundial de Salud Escolar ${ }^{(4)}$ realizada en el país a adolescentes de 13 a 15 años, el sobrepeso aumentó del $24,5 \%$ al $28,6 \%$ y la obesidad pasó del $4,4 \%$ al 5,9\% en comparación con los datos de la misma encuesta realizada cinco años antes. Además, con respecto a los hábitos alimentarios, se detectó que solo el $17,6 \%$ de los encuestados consumía cinco porciones diarias de frutas y verduras, y el $48,1 \%$ consumía dos o más bebidas azucaradas por día.

Los niños y niñas con sobrepeso u obesidad tienen mayor riesgo de padecer enfermedades no transmisibles ${ }^{(5,6)}$. En consecuencia, tienen mayor probabilidad de muerte y discapacidad prematura en la edad adulta. Entre las principales consecuencias de la obesidad en la infancia y la adolescencia se encuentran el incremento de los factores de riesgo de enfermedades cardiovasculares (como el colesterol elevado y la hipertensión) ${ }^{(6)}$ y el mayor riesgo de padecer apneas de sueño, problemas del aparato locomotor y problemas sociales $y$ psicológicos ${ }^{(7,8)}$.

Existe evidencia que señala que la publicidad de alimentos tiene un efecto sobre las preferencias, conducta de compra y patrones de consumo de los niños y las niñas. Al mismo tiempo, hay estudios que dan cuenta de una asociación entre la frecuencia de exposición a publicidades de alimentos poco saludables en la televisión y el consumo de los alimentos publicitados ${ }^{(9)}$. Estudios realizados en el país señalan que los alimentos que se promocionan en las publicidades dirigidas a niños y niñas presentan, en la mayor parte de los casos, una baja calidad nutricional ${ }^{(10)}$.

La relación entre exposición a la publicidad y sobrepeso/obesidad se explica por diversas razones, entre las cuales se encuentran el aumento del consumo de los productos publicitados y la influencia de niños y niñas en las decisiones de compra de las madres ${ }^{(11)}$.

Resulta importante examinar el papel de las madres en la mediación del consumo de alimentos en niños y niñas ya que ellas son los principales agentes socializadores en los hábitos y preferencias alimenticias. Asimismo, las madres son quienes funcionan como guardianes, ya que median en el proceso de selección de los alimentos y deciden en última instancia qué comprar o no ${ }^{(12)}$. La alimentación se entiende como una práctica socialmente situada que implica prácticas estandarizadas y saberes compartidos ${ }^{(13)}$. El proceso de toma de decisiones que supone la planificación de la alimentación de la familia es considerado un proceso complejo en el que intervienen factores biológicos, ecológicos y demográficos, políticos, sociales y culturales ${ }^{(14)}$.

En la actualidad, las prácticas vinculadas a la alimentación se encuentran inmersas en un sistema social, en el que rige la lógica del mercado como principio rector $^{(15)}$. A partir de comprender la alimentación como una práctica social que se desarrolla en un contexto histórico determinado es que nos interrogamos acerca del impacto de la publicidad en las experiencias y percepciones 
de las madres, quienes tienen un rol fundamental en las decisiones de compra respecto a los alimentos que se consumen y en los hábitos saludables que se desarrollan en el hogar ${ }^{(16,17)}$.

En este trabajo, el concepto de publicidad se entiende como "cualquier forma pagada de presentación y promoción no personal de ideas, bienes o servicios por un patrocinador identificado" y la promoción como "incentivos a corto plazo que fomentan la compra o venta de un producto o servicio"(18).

El presente estudio forma parte de una investigación multi-componente sobre la publicidad de alimentos dirigida a niños y niñas de la Argentina cuyo fin es promover políticas para reducir la obesidad infantil. La investigación posee cuatro componentes: a) un análisis del marco regulatorio actual, que señala los principales actores involucrados en la regulación de la publicidad de alimentos y analiza los potenciales obstáculos, desafíos y facilitadores en la promoción de cambios regulatorios en la Argentina ${ }^{(19)}$; b) un análisis cuantitativo de la cantidad y características de las publicidades de alimentos dirigidas a niños y niñas en la televisión argentina ${ }^{(10)}$; y c) un análisis nutricional de los alimentos publicitados $^{(10)}$. En este trabajo se presentan los resultados del cuarto componente de la investigación, el cual tiene como objetivo analizar cómo las madres de diferentes niveles educativos perciben la publicidad de alimentos dirigida a niños y niñas, señalando cómo esta influye en las demandas de compra de sus hijos y en las estrategias que estas desarrollan para responder a esas demandas. Asimismo, se analiza cómo la publicidad de alimentos se combina con otros elementos del contexto que afectan el proceso de toma de decisiones respecto a la alimentación de sus hijos y se instalan determinados consumos de productos no saludables. En el presente trabajo aportaremos información para comprender cómo la publicidad y promoción de alimentos dirigida a niños y niñas afectan las decisiones de compra de las madres respecto a la alimentación de sus hijos, identificando actitudes diversas según nivel educativo. Asimismo, el artículo se propone identificar otros factores que inciden en este proceso de toma de decisiones y aportar información para la regulación de la publicidad de alimentos infantiles.

\section{MATERIALES Y MÉTODOS}

La presente investigación es un estudio descriptivo de corte transversal que utiliza metodología cualitativa.

La población objetivo fueron madres de niños y niñas de entre 5 y 13 años. Se priorizó la incorporación de las madres porque la evidencia señala la importancia de estas en la organización de la alimentación del hogar ${ }^{(20,21)}$. Se consideró el tramo etario de 5 a 13 años porque incluye a niños que ya han desarrollado sus habilidades orales, de forma que pueden solicitar verbalmente los alimentos a sus padres y coincide con la escolaridad primaria en nuestro país. Asimismo, se definió la niñez, en términos demográficos, como el período que abarca hasta los 14 años de edad ${ }^{(22)}$. Para evitar sesgos, se excluyeron aquellas madres que fueran profesionales de la salud (médicas, nutricionistas) o ejercieran profesiones relacionadas a temas nutricionales. Se seleccionaron madres de diferente nivel educativo, ya que el nivel educativo de los padres constituye un proxy del nivel socio económico(23), a la vez que se encuentra asociado a la situación nutricional de los niños ${ }^{(24)}$. Los grupos focales se desarrollaron entre julio y noviembre del 2015. Todas las madres incluidas en el estudio residían en el Área Metropolitana de Buenos Aires (AMBA), área geográfica que incluye la Ciudad Autónoma de Buenos Aires, más 34 partidos de la provincia de Buenos Aires.

El Comité de Ética en Protocolos de Investigación (CEPI) del Hospital Italiano de Buenos Aires evaluó y aprobó el presente estudio (código de aprobación 2115). Todos los participantes firmaron el consentimiento informado. Se garantizaron la confidencialidad y el anonimato de los datos. Se utilizan nombres ficticios para identificar a los participantes. 
Se utilizaron grupos focales como técnica de recolección de datos. La técnica de grupo focal se encuadra dentro de las técnicas de recolección cualitativas y se define como un tipo de entrevista grupal en la que un grupo pequeño de individuos se junta con el propósito de discutir uno (o más) temas de interés, en este caso, la importancia que posee la publicidad de alimentos en la toma de decisiones respecto a la alimentación de sus hijos. Las participantes de los grupos fueron reclutadas a través del mecanismo de bola de nieve y de directivos de escuelas. Se diseñó una guía de entrevista con pocas preguntas, las cuales fueron desarrolladas por el equipo de investigación sobre la base de la revisión de la bibliografía sobre el tema y los objetivos de la investigación. En orden a analizar la claridad, comprensión y pertinencia de las preguntas, se realizó un estudio piloto con un grupo focal de cuatro madres y padres de niños y niñas de 5 a 12 años. El estudio piloto permitió responder las siguientes preguntas: ¿es claro el significado de la pregunta?, ¿las preguntas usan terminología que es comprensible para los participantes?, ¿las preguntas indagan solo sobre un tema a la vez?, ¿las preguntas tienen algún tipo de sesgo o guían a una respuesta determinada? Luego del grupo focal realizado se ajustó el instrumento de recolección de datos.

Los grupos focales, realizados entre mayo y noviembre de 2015, fueron conducidos por dos profesionales del equipo de investigación y tuvieron una duración aproximada de una hora. Los principales ejes de indagación de las entrevistas fueron: la importancia de la publicidad en la demanda de los niños y las respuestas de las madres (¿qué alimentos solicitan los niños?, ¿se accede a estos pedidos?, ¿por qué razones se solicitan estos productos?, etc.); percepciones respecto del contenido de las publicidades (¿qué publicidades de alimentos recuerda?, ¿cuáles resultan más atractivas para los niños?, ¿qué tipo de alimentos cree que se publicita?); percepciones respecto a la alimentación de los niños (¿cómo cree que se alimentan los niños actualmente?, ¿qué factores contribuyen a la alimentación de los niños?). Asimismo, durante el grupo focal se exhibió una publicidad y se discutió acerca del producto promocionado y la técnica publicitaria utilizada. En el desarrollo de los grupos focales se tuvo en cuenta una posible limitación de este instrumento de recolección vinculado a la respuesta "teórica". En una situación artificial como aquella en la que se desarrolla la recolección de datos, los entrevistados pueden adoptar una postura igualmente artificial, en este caso la de la madre preocupada por el tipo de alimentos publicitados. En la moderación del grupo se intentó evitar este tipo de respuestas y, para impedir estos sesgos por "conveniencia social" (social desirability bias) se preguntó a las madres no tanto por su propia experiencia sino en tanto "representantes" de un grupo poblacional.

El contenido de los grupos focales fue grabado y transcripto verbatim y analizado junto con las notas tomadas por los dos investigadores presentes en los grupos. Para analizar los datos se desarrollaron códigos que surgieron de forma inductiva durante el proceso de codificación, y luego se agruparon en dimensiones y categorías. Para el análisis de los datos se utilizó el software Atlas.ti 7.0.77. En el análisis de los datos se tuvieron en cuenta tanto las particularidades de los grupos focales, como el contexto de la conversación en donde se desarrollaban los Comentarios a fin de identificar ambigüedades, inconsistencias y los temas que se percibían con mayor o menor atención. La codificación preliminar de los datos fue realizada por un investigador y luego socializada con otro miembro del equipo. Sobre la base de la discusión, se revisaron los códigos y temas identificados y las definiciones adoptadas. Asimismo, en el análisis se identificaron el consenso alcanzado entre las diferentes opiniones y las diferencias existentes entre las madres de distintos niveles educativos.

\section{RESULTADOS}

Se realizaron ocho grupos focales con un total de 49 madres de diferente nivel educativo y con al menos un hijo o hija de 
Tabla 1. Cantidad y características de las participantes de los grupos focales. Área Metropolitana de Buenos Aires, Argentina, 2015.

\begin{tabular}{lccl} 
Grupo & Cantidad & $\begin{array}{c}\text { Edad } \\
\text { promedio }\end{array}$ & Nivel educativo \\
\hline Grupo focal 1 & 5 & 36,4 & Secundario incompleto \\
Grupo focal 2 & 4 & 28,7 & Secundario incompleto \\
Grupo focal 8 & 5 & 28,6 & Secundario incompleto \\
Grupo focal 3 & 12 & 38,0 & Secundario completo \\
Grupo focal 6 & 4 & 40,2 & Secundario completo \\
Grupo focal 4 & 7 & 41,0 & Secundario completo \\
Grupo focal 5 & 7 & 35,1 & Universitario completo \\
Grupo focal 7 & 5 & 36,0 & Universitario completo \\
\hline Fuente: Elaboración propia. & & \\
\hline
\end{tabular}

5 a 13 años (Tabla 1). El análisis inductivo de los datos permitió identificar tres grandes temas: 1) percepción sobre los efectos de la publicidad: demandas y estrategias para responderlas; 2) percepción de la influencia del contexto en la selección de alimentos; y 3) percepción acerca de los consumos "inevitables".

\section{Percepción sobre los efectos de la publicidad: demandas y estrategias para responderlas}

\section{Las demandas}

En los diferentes grupos realizados se ha encontrado un consenso respecto a la influencia de la publicidad en los deseos de compras y en las demandas de los niños. Si bien en un primer momento del grupo focal, al indagar sobre los pedidos de los niños en general, no se señala claramente la importancia de la publicidad y la promoción, a lo largo de la conversación este tema surge de modo recurrente en los distintos grupos y se comparten distintas experiencias que dan cuenta de la influencia de la publicidad en las demandas. En la discusión identifican que los pedidos están motivados no solo por el sabor del producto ("piden porque les gusta"), sino especialmente por la publicidad, ya que muchas veces se solicitan productos que no han probado con anterioridad.

\section{Los chicos quieren lo que ven en tele, eso es lo que les llama la atención. \\ (Fernanda, grupo focal 3)}

Las madres perciben a los niños como demandantes activos de diferentes tipos de alimentos. El supermercado o la salida del colegio son los lugares donde más se observan esos pedidos. Se señala la insistencia del "comprame, comprame, comprame", en especial en los niños más pequeños (de 5 años aproximadamente).

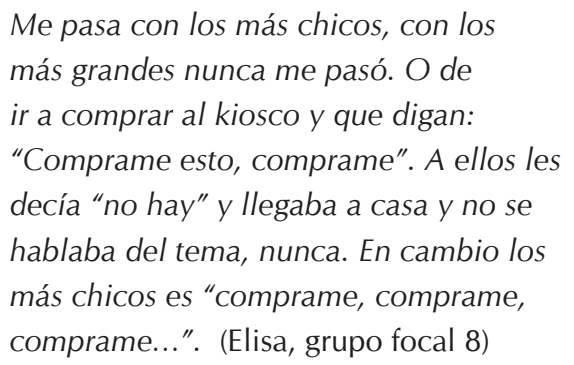

Las participantes destacan que la publicidad en televisión ha aumentado a lo largo del tiempo y que es la que "les calienta la cabeza a los chicos". Los niños no solo piden los productos que ven en las publicidades de la televisión sino también en las publicidades y promociones que ven en los supermercados y espacios públicos. Según las participantes, los niños se encuentran "saturados de publicidad". Se señala cómo el pedido del producto se realiza por el "premio" o "el personaje". El alimento resulta accesorio, aquello que rodea al producto es lo que incentiva el consumo.

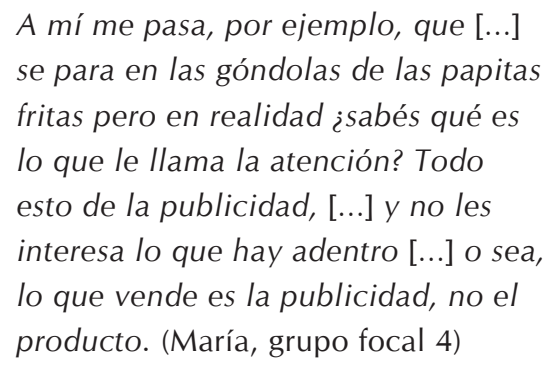


La aparición de personajes en la publicidad o en el paquete, y los regalos y promociones asociados a la compra del producto, son las principales razones que impulsan el pedido en los niños, según consideran las madres. Si bien las madres mencionan "la publicidad" de forma general, sin distinguir entre canales y técnicas de marketing empleadas, señalan la técnica publicitaria que incluye el uso del "personaje", el "muñeco", ya sea de una marca en particular o personajes de alguna película como las técnicas más efectivas para impulsar el consumo de niños y niñas. Este aspecto ha sido señalado en reiteradas ocasiones en todos los grupos realizados.

[Su hija] está comiendo el yogur por los dibujitos de Pachorra, que viene con cereales que antes no le gustaban y ahora le gustan por eso (por Pachorra). (Laura, grupo focal 5)

Entre las principales razones por las que los niños demandan los productos, además de los personajes, dibujitos y promociones, se señala la importancia del mensaje de las publicidades. Las madres relatan cómo los niños se sienten interpelados por el mensaje asociado al consumo del producto que se publicita. Las madres también refieren ser influenciadas por el mensaje de las publicidades, ya que este no solo interpela a los niños, sino que, en ocasiones, se dirige a los cuidadores, al resaltar las cualidades nutritivas de un determinado producto. El consumo se interrumpe en el caso de que un portavoz autorizado indique que la información de la publicidad no refleja la realidad del producto.

\section{Si no le dabas a tu hijo no eras buena madre, no te ocupabas de la salud de tu hijo porque no le dabas Danonino. Era un poco así la publicidad. ¿Se acuerdan? (Lorena, grupo focal 3)}

Con relación al producto nombrado en el testimonio anterior, una de las madres señala:
Yo me acuerdo que cuando eran chicas consumían Danonino pero porque era el auge de que crecían mejor y todo. Pero después el pediatra me dijo "olvidate". (Luciana, grupo focal 3)

Asimismo, se identifica la demanda de marcas específicas. El reconocimiento de marcas por parte de los niños y la fidelidad hacia esas marcas es percibido por las madres como una actitud de sus hijos, pero son también las propias madres quienes privilegian la compra de determinadas marcas por sobre otras. A diferencia de los aspectos previamente señalados, en los que existía un consenso en todos los grupos respecto a cómo los personajes y promociones impulsan la demanda de ciertos productos, no ha existido un consenso en los grupos con relación a las marcas: mientras algunas madres señalan que realizan sus compras buscando marcas particulares, otras dicen no considerarlas $y$, aun cuando consumen los productos publicitados, sustituyen las marcas publicitadas por otras de menor precio u otras que consideran más saludables.

Sí, totalmente. Yo con los cereales ya hice eso, yo no compro cereales, los compro sueltos, los compro en una dietética. $O$ sea, no le compro cereal de caja. A ella le encanta por ahí el muñequito de Nesquik y qué sé yo, pero esos cereales ella sabe que en casa no va a haber por varios motivos, porque tienen más azúcar y porque son más baratos los otros también, o sea, son más sanos y más baratos. (Nora, grupo focal 4)

En los grupos focales de madres de menor nivel educativo se observa que se enfatiza la importancia de la marca al momento de realizar las compras. Estas madres señalan como positivo el acceso a determinadas marcas y la homologación de ciertas marcas con lo "bueno".

Sí, también cuando ya conocés la marca decís: "Sí, ya sé, la conozco, es buena, seguro que lo que salga va a ser bueno". (Verónica, grupo focal 8) 


\section{Acceder a las demandas: razones y estrategias}

Ante las demandas de los niños, influenciadas por la publicidad de alimentos, las madres manifiestan diferentes actitudes que se vinculan al manejo de los límites al interior del hogar. La cuestión de los límites excede a los alimentos, y se vincula a "decir que no" a los pedidos de los niños. Respecto de los límites que se establecen en el consumo de ciertos alimentos, existen diversas posturas que abarcan desde aquellas madres que establecen límites claros y dicen no verse influenciadas por los pedidos de los niños, hasta aquellas que encuentran dificultades para poder "decir que no". "Soy medio obse", "soy un desastre" son diferentes calificativos que utilizan las entrevistadas para definir su actitud frente a la educación de los hijos, en general, y a la comida, en particular. Las diferentes actitudes que toman las madres median entre las demandas de los niños y sus propias decisiones de compra. La negativa a consumir determinados productos por su calidad nutricional solo se ha observado en algunos miembros de los grupos de mayor nivel educativo, en especial respecto de las bebidas azucaradas y ciertos alimentos considerados "malos" (salchichas, hamburguesas, etc.).

Yo soy bastante hinchapelotas. Ponele, a las papas fritas, todo eso, yo no le compro, nada de eso, en la calle. En verano sí puedo comprarle un helado, esas cosas, por ahí puede suceder o que sabemos que no hay nada a la noche y me dice: "Ay, compremos un chocolatito hoy". (Nora, grupo focal 4)

Me he encontrado con los gritos de él pidiendo algo [...] en el supermercado. Entonces, lo que no me gusta o que trato de controlar es la conducta, que es no solo con la comida sino en general, de manipularme y de hacer un escándalo. (Graciela, grupo focal 7)

Las madres perciben que cuando los niños toman contacto directo con los productos en las góndolas de los supermercados o en los kioscos, las demandas se intensifican. Es decir, señalan que los pedidos de compra son más insistentes en los momentos en que los niños se encuentran frente al producto. Ante estas demandas, las madres adoptan diferentes respuestas que abarcan desde ceder ante la demanda para que "pruebe", la negativa a la compra, o no ir con los niños al supermercado para evitar la demanda. Las madres manifiestan terminar accediendo a la compra de determinados productos ante la insistencia de los niños y para "evitar el berrinche". El consumo del producto se da como una respuesta frente a la insistencia de los niños, en especial en los supermercados.

\section{$A$ veces hay que pasar por esa prueba de que no les gusta y decirle: "mirá: no te gustó, no te lo compro más", entonces ahí es como que una está autorizada a no com- prarle porque ¿qué otro motivo tenés para no comprarle? Es como que no le podés decir que no porque sí. (Ana, grupo focal 6) \\ Lo que pasa es que si no te tenés que venir con un berrinche de allá [el super- mercado] hasta tu casa y prefiero a veces, "tomá"... (Sarah, grupo focal 3)}

Las participantes señalan que el consumo de ciertos alimentos "se negocia" y se establece una frecuencia para aquellos que no se consideran saludables. Los que se negocian son aquellos productos considerados "porquerías": las gaseosas, en algunos casos, y los snacks. Las madres intentan negociar entre aquello que saben que es ideal no consumir y lo que realmente pueden llevar a cabo. De esta forma, restringen el consumo del producto, pero no lo eliminan de sus compras.

No, le digo, porquerías ya comiste: comiste el lunes. No, le digo, ya tuvimos la cuota cubierta de porquerías de la semana. (Julia, grupo focal 4)

Y siempre también me piden galletitas dulces, por ahí, para tomar la leche y trato de que no sea todos los días... (Irma, grupo focal 7) 
La compra de alimentos o la salida a restaurantes de comida rápida también es utilizada por las madres como "premio" o "castigo". De esta forma, las razones por las que las madres acceden a las demandas de los niños dan cuenta de distintos significados de las prácticas vinculadas a la alimentación y el consumo de ciertos productos que exceden las cuestiones nutricionales.

Yo las uso mucho para extorsionarlas [risas]. Comprar porque sí, no. Si los voy a comprar les voy a sacar el jugo de alguna manera. Bueno, ahí como premio de algo, si no, no. Trato de no comprarlos. (Irma, grupo focal 7)

Bueno, los Shimmy que les gustan tanto, cuando voy al súper les digo: "Si me dejaron comprar tranquila, se portaron bien, al final agarramos uno y nos vamos con eso, si no, no". Con eso también los tengo controlados. (Marta, grupo focal 7)

\section{Percepción acerca de la oferta de alimentos y su consumo: cambios en el tiempo}

Las madres han identificado distintos elementos del contexto que consideran que afectan el tipo de alimentación de los niños y que se combinan con el efecto de la publicidad. Al comparar con otras épocas, se señala que la actualidad está caracterizada por la "falta de tiempo" y esto se relaciona con el consumo de ciertos tipos de productos (hamburguesas, salchichas, etc.). En las discusiones respecto a la publicidad de alimentos existe un consenso en los diferentes grupos acerca de que los alimentos publicitados son "malos" o "no tan buenos" y dentro de este conjunto de alimentos se identifican claramente ciertos productos: salchichas, hamburguesas y otros productos considerados como "porquerías". Las razones para su consumo se relacionan con el "tiempo" y la "rapidez" para cocinarlos. Estos consumos se dan en parte por la demanda de los niños, pero también por decisión de las madres, quienes los seleccionan por las características enunciadas.
Coordinador: ¿Qué serían los alimentos malos?

Fabiola: Salchichas.

Blanca: Patitas.

Soledad: Claro, me parece que las golosinas y todo eso.

Ana: No sé si "malo" o "no tan bueno". No buenos por lo menos.

Blanca: Hay chicos que...

Fabiola: En mi casa salchichas... no se compran ni salchichas, ni patitas de pollo. Salvo un día, hay un cumpleaños, un evento que vienen muchos niños a casa y quedó la bolsa. Si no, patitas, salchichas y hamburguesas tampoco les gusta mucho. Soledad: Yo hamburguesas tampoco, a mí la hamburguesa me da pánico. Pero el resto, la verdad, que no... yo no lo tengo como habitual pero sí a mí me zafa.

Ana: Sí, yo las patitas también tengo por las dudas.

Soledad: Me parece que me he vuelto un desastre.

Blanca: Yo al grande le tengo guardados siempre patitas porque el grande por ahí te cae con dos o tres amigos y... (Grupo focal 5)

Se señala que, actualmente, hay una mayor oferta y variedad de productos. Sin embargo, la percepción acerca de esta situación no es uniforme en todos los grupos. Mientras aquellas madres de mayor nivel educativo observan con cierta preocupación el aumento de la oferta de alimentos, entre las madres de los grupos focales de menor nivel educativo, la mayor oferta de productos es percibida por muchas de ellas como algo positivo, ya que se vincula a la posibilidad de tener acceso a productos a los que antes no accedían.

[Antes] era una Manon y punto y ahora hay como una variedad. O sea, aparte viene hasta el packaging para que pueda ser...O sea, transportado por los chicos y ponérselo en la mochila y entran justo en el espacio en el que va en la mochila. Me parece que cuando éramos chicos no había esa variedad. El estímulo ahora es muchísimo mayor, con la tele, todo... (Nora, grupo focal 4) 
Mi marido, por ejemplo, era más pobre que yo y dice que comía solamente a la noche cuando llegaba la madre que trabajaba todo el día y a veces le dice a las chicas, a Delfina: "tienen que cuidar y valorar lo que nosotros le damos porque yo me acuerdo que ni una Coca Cola tomábamos". Entonces uno a veces les da a los hijos lo que uno... lo que no nos dieron a nosotros. (Cora, grupo focal 6)

La mayor oferta de alimentos se combina con "niños más demandantes". Las madres señalan cómo los niños poseen en la actualidad un rol más activo en las demandas y cómo estas son más tenidas en cuenta por las madres. Son los propios niños quienes seleccionan los alimentos. Esto es mencionado por igual en todos los grupos focales realizados.

Yo lo que veo ahora, digamos, que los chicos tienen poder de decisión en la cocina: "Quiero comer esto, quiero comer aquello". Yo me acuerdo, en mi época de chica, me sentaban y tenías que comer eso. (Amalia, grupo focal 4)

Si bien en distintos grupos varias madres acuerdan en que en la actualidad la alimentación de los niños es "peor" que en otras épocas, en las madres de mayor nivel educativo se menciona, en algunas ocasiones, el hecho de que actualmente las madres están más concientizadas respecto a la alimentación de sus hijos y que, por esa razón, eligen opciones más saludables.

En realidad, a mí se me juega una doble... yo creo que en mi caso en particular, me parece que hoy por hoy, insisto, en mi caso, hay más ofertas pero yo creo que también hoy por hoy hay más conocimiento. Me parece, qué sé yo, también al niño se le da como un lugar distinto, de estar más atento que coma mejor, no sé si todo el mundo. (Sarah, grupo focal 5)

\section{Los consumos percibidos como inevitables: las bebidas azucaradas y los restaurantes de comida rápida}

En los diferentes grupos se mencionan algunos consumos por parte de niños y niñas y la familia que están incorporados al consumo cotidiano, a pesar de ser reconocidos por las madres como alimentos de baja calidad nutricional. Las salidas a restaurantes de comida rápida, por ejemplo, tienen un significado más amplio que el consumo de los productos que allí se ofrecen. Se percibe como un paseo y un lugar donde los niños se entretienen. Asimismo, se observan las cuestiones anteriormente mencionadas, como la importancia de las promociones, en el caso de los juguetes asociados a la compra de productos, y los alimentos como "premios". En los grupos focales de madres de menor nivel educativo se encuentra menos problematizado el consumo en restaurantes de comida rápida y se identifican menos restricciones con respecto a este consumo.

Cobro la pensión y seguro, cuando cobro, dos veces al mes la llevo. Porque está la otra también que tiene 12 años, es grandota pero tiene mente de criatura, que también quiere ir a McDonald, quiere comer hamburguesas. (Mirta, grupo focal 1)

Por ejemplo, si me traés una buena nota te dejo, te doy plata para ir a McDonald. O sale de rugby, va con un amigo, si me trae una buena nota. Si no, no. (Lucía, grupo focal 3)

Entre los alimentos que más se demandan y negocian para su consumo, las gaseosas azucaradas son las más mencionadas. En algunos casos, su consumo se reserva para ciertas ocasiones, pero raramente se restringe por completo. En los diferentes grupos realizados, se observan diferentes actitudes: parecería darse un mayor consumo en los grupos de menor nivel educativo y mayor problematización acerca del consumo en los grupos de nivel educativo más alto. Se señala que, aun cuando no se consuma en el hogar, una vez 
que los niños reconocen el sabor comienzan a demandarlo.

\author{
Los cumpleaños, lo social hace que ellos \\ empiecen a probar cosas que por ahí \\ van más allá de lo que uno los deja o \\ no los deja, quiere o no quiere. No sé, \\ me acuerdo que en los cumpleaños, yo \\ no fui a comprarle la Coca Cola pero \\ después no hubo vuelta atrás: "La Coca \\ Cola, la Coca Cola...". Lo mismo los \\ snacks... (Fabiana, grupo focal 7)
}

\section{DISCUSIÓN}

Este estudio contribuye al cuerpo de investigaciones que señalan que las publicidades y promociones son un factor a considerar en el momento de comprender las prácticas alimenticias de los niños ${ }^{(9,25)}$. Investigaciones previas ${ }^{(26,27)}$ muestran que los niños tienen una influencia cada vez mayor sobre la compra y el consumo de alimentos y una de las razones que pueden explicar esta influencia es el denominado "efecto fastidio" que da cuenta de la tendencia de los niños a solicitar en forma insistente los productos que se publicitan y promocionan. El presente estudio coincide con estos trabajos al mostrar cómo los personajes en el envase y en las publicidades, junto a las promociones, disparan la demanda de los niños ${ }^{(26)}$ y cómo esta situación se intensifica en el momento en que los niños toman contacto con el producto, tanto en salidas en el supermercado como en los kioscos.

El estudio muestra que el "efecto fastidio" no es la única razón por la cual las madres acceden a los pedidos de los niños. Tal como ha sido señalado en otra investigación ${ }^{(28)}$, las madres pueden utilizar la alimentación de su hijo no solo como una forma de proveerle los nutrientes necesarios, sino también como una forma de brindar recompensas o premios. Se da cuenta así de los diferentes significados que se le otorga a la alimentación y cómo esta trasciende la mera satisfacción de una necesidad básica. En el trabajo se observó cómo la alimentación y, en este caso en particular, la compra de determinados productos alimenticios demandados por niños y niñas no solo están asociadas a una recompensa, sino también a una forma de expresión de cariño materno/familiar hacia el niño, o de festejo de un acontecimiento importante.

Las madres consideran que sus elecciones de compra se basan en sus decisiones individuales. Esto debe considerarse en consonancia con el discurso que se propone desde las empresas de alimentos ultraprocesados, las que alientan la idea de que la alimentación es una responsabilidad exclusiva del individuo ${ }^{(29)}$. Este dato es coincidente con estudios realizados en otros países que muestran que la obesidad es percibida como el resultado de conductas individuales y no como la responsabilidad de las prácticas de la industria de alimentos y de la falta de regulación por parte del Estado ${ }^{(30)}$. Esta situación plantea un desafío y una necesidad para los actores vinculados a la salud pública de sensibilizar a la población respecto a la influencia del entorno, en general, y de la publicidad de alimentos, en particular, en el sobrepeso y la obesidad, especialmente de niños y niñas, y de la necesidad de diseñar, promover e implementar políticas públicas que reduzcan la exposición de los menores a la publicidad de alimentos no saludables ${ }^{(1)}$.

En los grupos se reconocen distintos elementos del entorno que han modificado las conductas alimentarias a lo largo del tiempo como la mayor oferta de alimentos, y se señala que los estilos de vida actuales llevan a tomar determinadas decisiones que consideran que no son las ideales, como preparar comidas rápidas, por ejemplo, en un contexto donde los niños "son más demandantes" y hay una mayor oferta de productos ultraprocesados (snacks, galletitas, bebidas gaseosas, cereales endulzados para el desayuno, yogures endulzados, hamburguesas, salchichas, etc.). La mención de niños "más demandantes" supone la construcción de un/a niño/a como consumidor/a, lo que da cuenta de cómo la lógica del mercado interpela desde tempranas edades a los sujetos 
y afecta las prácticas alimentarias ${ }^{(15)}$. La presencia de estos/as niños/as se combina con madres que no "se sienten autorizadas a enseñar a sus hijos a comer"(31). En este contexto, los mensajes legitimados por los medios de comunicación y las demandas de los niños se vuelven principios rectores de la alimentación. De este modo, resulta entendible que, a lo largo de las discusiones que se han dado en los grupos focales, las madres se muestren confundidas respecto a la calidad nutricional de los productos que consumen.

Los resultados de la investigación son coincidentes con aquellos que señalan que el conocimiento respecto a la calidad nutricional no predice por sí solo el comportamiento alimentario ${ }^{(14)}$. Si bien hay consenso, en todos los grupos focales realizados, en cuanto a la existencia de alimentos "malos", que son los más publicitados, y en que los alimentos ultraprocesados son "peores" que la comida casera, se señala también que se consumen estos alimentos porque su consumo "es más fácil", y "se hacen rápido".

Un hallazgo importante de la investigación es la actitud diferencial hacia la publicidad de las madres de diferentes niveles educativos. En las madres de mayor nivel educativo se observa una mayor sensibilización respecto al consumo de ciertos tipos de alimentos, como bebidas azucaradas y alimentos ultraprocesados como salchichas, hamburguesas, cereales, etc. Si bien esto se ha observado en algunas madres de estos sectores, resulta preocupante que en los sectores de menor educación parecería existir una menor problematización y sensibilización al respecto. Mientras que en los sectores de mayor nivel educativo se identifican estrategias para evitar determinados alimentos y reemplazarlos por productos más saludables, las madres de menor nivel educativo, si bien destacan la importancia de la comida casera, poseen una mirada menos crítica respecto a la amplia oferta de productos ultraprocesados y el mayor acceso a este tipo de productos. Es interesante observar que el consumo de determinados productos se menciona como algo aspiracional, y se destaca la importancia de consumir determinadas marcas y acceder a determinados productos a los que "antes no se podía" acceder. En este sentido, resulta relevante comprender la práctica de la alimentación dentro de un sistema de valores y un contexto determinado en los que distintos grupos sociales otorgan distintos significados al "comer bien"(17). El consumo de productos ultraprocesados no debe entenderse simplemente como desconocimiento o aspiración, sino que debe comprenderse en un contexto social determinado, donde las mujeres se enfrentan a una serie de exigencias vinculadas al trabajo doméstico y extradoméstico, donde dicho consumo permite satisfacer demandas de los niños y también ahorrar tiempo (les permite "zafar"). Estas circunstancias son explotadas desde la publicidad, en la que se alienta y legitima el consumo de productos procesados y de fácil preparación.

Resulta relevante considerar el conjunto de relaciones sociales que afectan la capacidad de "elegir" de las distintas madres. Las restricciones en los ingresos y en el tiempo disponible para realizar las tareas vinculadas a la alimentación pueden funcionar como barreras para elegir opciones más saludables. Esto debe considerarse especialmente en el caso de las mujeres de menores ingresos, ya que son quienes poseen una mayor participación en las tareas de cuidado debido a la imposibilidad de delegar estas actividades ${ }^{(32)}$.

Las diferencias entre sectores socioeconómicos deben ser consideradas para el diseño de políticas efectivas que logren disminuir las brechas en la prevalencia de la obesidad en la población infanto-juvenil. Este aspecto resulta de especial relevancia porque el sobrepeso y la obesidad afectan en mayor medida a los sectores más vulnerables ${ }^{(33,34)}$.

En este contexto, es importante resaltar el lugar del Estado en el diseño de una política alimentaria que incluya a distintos sectores comprometidos con la salud de la población y permita mejorar el entorno alimentario y "no dejar en manos del mercado la única lógica de la cadena"(15). Nuestros resultados resaltan la necesidad de diseñar políticas que desincentiven el consumo de alimentos de baja calidad nutricional. Entre estas políticas, la restricción de la publicidad 
de alimentos no saludables dirigida a niños se considera como un componente fundamental de cualquier estrategia integral que intente abordar este problema. En este sentido, la OMS elaboró el Plan de acción mundial para la prevención y el control de las enfermedades no transmisibles 2013$2020^{(35)}$ en el que propone la restricción de la publicidad de alimentos y bebidas no alcohólicas dirigida a niños, niñas y adolescentes. Y el último informe de la Comisión para acabar con la obesidad infantil también señala la necesidad de reducir la exposición a la publicidad de alimentos no saludables dirigida a niños y adolescentes ${ }^{(36)}$. En nuestra región, la Organización Panamericana de la Salud aprobó el Plan de Acción para la prevención de la obesidad en la niñez y en la adolescencia $^{(37)}$ en el que destaca la regulación de la publicidad como una línea de acción estratégica para la región. Una política efectiva de restricción de la publicidad de alimentos no saludables dirigida a niños y niñas debe contar con los siguientes elementos: a) ser de carácter integral, de manera de incluir no solo los medios y canales de comunicación tradicionales sino también la publicidad no tradicional, como las estrategias de marketing relacional; $y$ b) establecer a través de perfiles nutricionales el carácter saludable del alimento ${ }^{(38)}$.

El presente estudio representa un análisis novedoso en el país acerca de la percepción de las madres sobre cómo la publicidad afecta la alimentación de niños y niñas, identificando distintos elementos del entorno que impulsan el consumo de determinados productos. Las diferencias identificadas entre distintos grupos han mostrado la importancia de tener en cuenta la disparidad de ingresos al diseñar políticas de alimentación. Una limitación del presente estudio se encuentra vinculada al uso de grupos focales como herramienta para analizar las percepciones sobre cuestiones vinculadas a la alimentación, ya que las madres pueden haberse visto influidas en sus opiniones por el resto del grupo. Sin embargo, la dinámica de las discusiones y la espontaneidad de las respuestas indican que esta limitación ha podido ser superada a lo largo de la investigación. Se han incluido madres de la región del AMBA y de distintos niveles educativos a fines de dar cuenta de la heterogeneidad de la situación. En futuras investigaciones sería interesante replicar el estudio en centros urbanos más pequeños para describir diversas situaciones que pueden darse en otras regiones del país. Asimismo, sería interesante desarrollar una encuesta poblacional que permita establecer las diferencias encontradas entre madres de diferente nivel educativo en una muestra con representatividad estadística a fines de contar con información que permita diseñar políticas efectivas para toda la sociedad. Asimismo, es importante realizar estudios que analicen en profundidad los hábitos alimentarios y el consumo de productos ultraprocesados en la población.

\section{AGRADECIMIENTOS}

Agradecemos al International Development Research Centre (IDRC) de Canadá que financió el proyecto titulado "Television food advertising to children in Argentina" (Proyect ID 107459). Asimismo agradecemos a todos los participantes del estudio por haber brindado su testimonio, a la Dra. Patricia Aguirre por sus valiosas contribuciones en el diseño e implementación del estudio y especialmente a la Dra. Verónica Schoj, directora ejecutiva de la Fundación InterAmericana del Corazón Argentina, por el apoyo e importantes aportes brindados a lo largo de la investigación.

\section{REFERENCIAS BIBLIOGRÁFICAS}

1. Organización Mundial de la Salud. Conjunto de recomendaciones sobre la promoción de alimentos y bebidas no alcohólicas dirigida a los niños. Ginebra: OMS; 2010.

2. Organización de las Naciones Unidas para la Alimentación y la Agricultura. Panorama Nutricional en América Latina y el Caribe. Roma: FAO; 2015.

3. Agurs-Collins T, Bouchard C. Gene-nutrition and gene-physical activity interactions in the etiology of obesity. Obesity (Silver Spring). 2008;16(Suppl 3):S2-S4. 
4. Ministerio de Salud. Encuesta Mundial de Salud Escolar: Resumen Ejecutivo de Argentina. Buenos Aires: Ministerio de Salud; 2013.

5. Guo SS, Chumlea WC. Tracking of body mass index in children in relation to overweight in adulthood. American Journal of Clinical Nutrition. 1999;70(1):145-148.

6. Freedman DS, Khan LK, Mei Z, Dietz WH, Srinivasan SR, Berenson GS. Relation of childhood height to obesity among adults: the Bogalusa Heart Study. Pediatrics. 2002;109(2):E23.

7. Daniels SR, Arnett DK, Eckel RH, Gidding SS, Hayman LL, Kumanyika S, Robinson TN, Scott BJ, St Jeor S, Williams CL. Overweight in children and adolescents pathophysiology, consequences, prevention, and treatment. Circulation. 2005;111(15):1999-2012.

8. Dietz WH. Overweight in childhood and adolescence. New England Journal of Medicine. 2004;350(9):855-856.

9. Hastings G, Stead M, McDermott L, Forsyth A, MacKintosh A, Rayner M, Godfrey C, Caraher M, Angus K. Review of research on the effecs of food promotion to children. Glasgow: Center for Social Marketing, University of Strathclyde; 2003.

10. Fundación Interamericana del Corazón Argentina. Informe de investigación: Publicidad de alimentos dirigida a niños y niñas en la TV argentina [Internet]. 2015 [citado 15 mar 2016]. Disponible en: http://tinyurl.com/z7uosfd.

11. Outley C, Taddese A. A content analysis of health and physical activity messages marketed to african american children during after-school television programming. Archives of Pediatrics and Adolescent Medicine. 2006;160(4):432-435.

12. O'Dougherty $M$, Story M, Stang J. Observations of parent-child co-shoppers in supermarkets: children's involvement in food selections, parental yielding, and refusal strategies. Journal of Nutrition Education and Behavior. 2006;38(3):183-188.

13. Warde A, Cheng SL, Olsen W, Southerton D. Changes in the practice of eating a comparative analysis of time-use. Acta Sociologica. 2007;50(4):363-385.

14. Nestle M, Wing R, Birch L, DiSogra L, Drewnowski A, Middleton S, Sigman-Grant M, Sobal J, Winston $\mathrm{M}$, Economos $\mathrm{C}$. Behavioral and social influences on food choice. Nutrition Reviews. 1998;56(5):50-64.
15. Aguirre P. Reflexiones sobre las nuevas formas del hambre en el siglo XXI: la obesidad de la escasez. Revista Sapiens Research. 2011;1(2):60-65.

16. Golan M, Crow S. Parents are key players in the prevention and treatment of weight-related problems. Nutrition Reviews. 2004;62(1):39-50.

17. Aguirre P. Ricos flacos y gordos pobres: la alimentación en crisis. Buenos Aires: Capital Intelectual; 2004.

18. Kotler P, Armstrong G. Fundamentos de marketing. México: Pearson Educación; 2003.

19. Fundación Interamericana del Corazon Argentina. Publicidad de alimentos dirigida a niños. Análisis y descripción del marco regulatorio [Internet]. 2016 [citado 15 mar 2016]. Disponible en: http://tinyurl.com/gtg773p.

20. Lahoz C. El papel clave de las mujeres en la seguridad alimentaria. En: Seguridad alimentaria y políticas de lucha contra el hambre: Seminario Internacional sobre Seguridad Alimentaria y Lucha contra el Hambre. Córdoba: Universidad de Córdoba; 2006. p. 117-129.

21. Franco-Patiño SM. La alimentación familiar: una expresión del cuidado no remunerado. Prácticas de oficio: Investigación y reflexión en Ciencias Sociales [Internet]. 2010;(6) [citado 15 mar 2016]. Disponible en: http://tinyurl.com/grljmm8.

22. Torrado S. Historia de la familia en la Argentina moderna (1870-2000). Buenos Aires: Ediciones de La Flor; 2003.

23. Galobardes B, Shaw M, Lawlor DA, Lynch JW, Smith GD. Indicators of socioeconomic position (part 1). Journal of Epidemiology and Community Health. 2006;60(1):7-12.

24. González-Jiménez E, Aguilar-Cordero MJ, García-García CJ, García-López P, Álvarez-Ferre J, Padilla-López CA, Ocete-Hita E. Influencia del entorno familiar en el desarrollo del sobrepeso y la obesidad en una población de escolares de Granada (España). Nutrición Hospitalaria. 2012;27(1):177-184.

25. Cairns G, Angus K, Hastings G, Caraher M. Systematic reviews of the evidence on the nature, extent and effects of food marketing to children: A retrospective summary. Appetite. 2013;62:209-215.

26. Henry HKM, Borzekowski DLG. The nag factor. Journal of Children and Media. 2011;5(3):298-317. 
27. McDermott L, O'Sullivan T, Stead M, Hastings G. International food advertising, pester power and its effects. International Journal of Advertising. 2006;25(4):513-539.

28. Marshall D, O'Donohoe S, Kline S. Families, food, and pester power: beyond the blame game? Journal of Consumer Behaviour. 2007;6(4):164-181.

29. Koplan JP, Brownell KD. Response of the food and beverage industry to the obesity threat. Jama. 2010;304(13):1487-1488.

30. Oliver JE, Lee T. Public Opinion and the Politics of America's Obesity Epidemic (KSG Working Paper No. RWP02-017)[Internet]. 2002 [citado 15 mar 2016]. Disponible en: http:// dx.doi.org/10.2139/ssrn.313824

31. Aguirre P, Díaz-Córdoba D, Polischer G. Cocina y comer en la Argentina de hoy. Buenos Aires: FUNDASAP, Fundación ESSEN; 2015.

32. Calero A, Dellavalle R, Zanino C. Uso del tiempo y economía del cuidado (Documento de Trabajo $\left.\mathrm{N}^{\circ} 09\right)$ [Internet]. 2015 [citado 15 mar 2016]. Disponible en: http://tinyurl.com/ z6da7e3.

33. Peña M, Bacallao J. La obesidad en la pobreza: un nuevo reto para la salud pública (Publicación Científica No 576). Washington DC: OPS; 2000.
34. Ponce $\mathrm{M}$, Allemandi L, Castronuovo L, Tiscornia V, Gutkowski P, Schoj V. Brechas sociales de la obesidad en la niñez y adolescencia: Análisis de la Encuesta Mundial de Salud Escolar (EMSE). Buenos Aires: Unicef, Fundación Interamericana del Corazón Argentina; 2016.

35. Organización Panamericana de la Salud, Organización Mundial de la Salud. Plan de acción mundial para la prevención y el control de las enfermedades no transmisibles 2013-2020. Washington DC: OPS, OMS; 2013.

36. World Health Organization. Report of the Commission on Ending Childhood Obesity. Geneva: WHO; 2016.

37. Organización Panamericana de la Salud, Organización Mundial de la Salud. Plan de acción para la prevención de la obesidad en la niñez y la adolescencia. Washington DC: OPS, OMS; 2014.

38. Fundación Interamericana del Corazón Argentina. Publicidad de alimentos dirigida a niños y niñas en la Argentina: Documento informativo [Internet]. 2016 [citado 15 mar 2016]. Disponible en: http://tinyurl.com/h9bqz6g. 